\title{
Some results concerning the Tremblay operator and some of its applications to certain analytic functions
}

\author{
Hüseyin Irmak \\ Department of Mathematics, \\ Faculty of Science, \\ Çankırı Karatekin University, \\ Çankırı, Turkey \\ email: hisimya@yahoo.com
}

\author{
Olga Engel \\ Szász Adalbert Sports High School, \\ Târgu Mureş, Romania \\ email: engel_olga@hotmail.com
}

\begin{abstract}
The aim of this scientific note is first to present certain information associated with the Tremblay operator in the complex plane and then to determine several results constituted by the related operator for certain analytic functions and also to point some implications of them out.
\end{abstract}

\section{Introduction, definitions and motivation}

As is known, in the literature, we often encounter many scientific studies in relation with fractional calculus that is that fractional integral or fractional derivative. They have important roles in both science and practice and are also used to define various new definitions or transformations. They or some of their applications naturally lead researchers to new studies or practices. In this respect, this study is one of the studies that we have indicated. Within this context and also in this present investigation, some new definitions and

2010 Mathematics Subject Classification: 26A33, 34A08, 35G10, 35F05, 30C55, 30C45, $30 \mathrm{~A} 10$

Key words and phrases: complex plane, unit open disk, normalized analytic function, fractional calculus, tremblay operator, equations and inequalities in the complex plane 
also applications of an operator, which is related to fractional calculus and known as the Tremblay operator in the literature, will be considered for certain functions with complex variable. For certain results relating to the Tremblay operator and also fractional calculus, one may check the paper in [1]-[3], [5], [7], [9]-[11], [15] and [16] in the references.

Let us now recall some definitions and information that will be relevant to our scientific research.

Firstly, let

$$
\mathbb{C}, \quad \mathbb{R}, \quad \mathbb{N} \text { and } \mathbb{U}
$$

be the set of complex numbers, be the set of real numbers and the set of positive integers and the unit open disk:

$$
\{z: z \in \mathbb{C} \text { and }|z|<1\} \text {, }
$$

respectively. Moreover, we denote by $\mathcal{A}(\mathrm{n})$ the family of the functions $\mathrm{f}(z)$ normalized by the following Taylor-Maclaurin series:

$$
f(z)=z+a_{n+1} z^{n+1}+a_{n+2} z^{n+2}+\cdots \quad\left(a_{n+1} \in \mathbb{C} ; n \in \mathbb{N}\right),
$$

which are analytic and univalent in $\mathbb{U}$.

We next recall, for a complex function $\kappa:=\kappa(z)$, the definition of fractional derivative is denoted by the following symbol:

$$
\mathcal{D}_{z}^{\mu}[\kappa] \equiv \mathcal{D}_{z}^{\mu}[\kappa(z)] \quad(0 \leq \mu<1)
$$

and also defined as in (cf., e.g., [1]-[3], [9]-[11], [14] and [15]):

Let $\mathrm{K}(z)$ be an analytic function in a simply-connected region of the $z$-plane containing the origin. Then, the fractional derivative of order $\mu$ is defined by

$$
\mathcal{D}_{z}^{\mu}[\kappa]=\frac{1}{\Gamma(1-\mu)} \frac{\mathrm{d}}{\mathrm{d} z} \int_{0}^{z} \frac{\kappa(\xi)}{(z-\xi)^{\mu}} \mathrm{d} \xi \quad(0 \leq \mu<1),
$$

where the multiplicity of $(z-\xi)^{-\mu}$ above is removed by requiring $\log (z-\xi)$ to be real when $z-\xi>0$. All right, here and throughout this paper, the notation $\Gamma$ denotes the well-known gamma function in the literature.

In the light of information above, for an analytic function $k(z)$, the wellknown derivative, which is the Srivastava-Owa derivative of order $m+\mu$, is then presented by

$$
\mathcal{D}_{z}^{\mathrm{m}+\mu}[\mathrm{k}] \equiv \frac{\mathrm{d}^{\mathrm{m}}}{\mathrm{d} z^{\mathrm{m}}}\left(\mathcal{D}_{z}^{\mu}[\mathrm{k}]\right) \quad(0 \leq \mu<1 ; \mathrm{m} \in \mathbb{N} \cup\{0\}),
$$


which readily yields

$$
\mathcal{D}_{z}^{0+\mu}[\kappa] \equiv \mathcal{D}_{z}^{\mu}[\kappa] \quad \text { and } \quad \mathcal{D}_{z}^{1+\mu}[\kappa] \equiv \frac{\mathrm{d}}{\mathrm{d} z}\left(\mathcal{D}_{z}^{\mu}[\mathrm{k}]\right) \quad(0 \leq \mu<1)
$$

Recently, by means of the Srivastava-Owa derivative [14], Tremblay [5] and [16], introduced and also studied an interesting fractional derivative operator:

$$
\mathcal{T}_{\tau, \mu}[\cdot] \quad(0<\tau \leq 1 ; 0<\mu \leq 1 ; 0 \leq \tau-\mu<1),
$$

which was defined in the domain of the complex plane and whose properties in several spaces were discussed systematically. For instance, one may refer to the works given by [5], [9]-[3] and [14]-[16].

For a function $\mathrm{f}(z)$ in $\mathcal{A}(\mathrm{n})$, the Trambley operator:

$$
\mathcal{T}_{\tau, \mu}[\mathrm{f}] \quad \text { or } \quad \mathcal{T}_{\tau, \mu}[\mathrm{f}(z)]
$$

is defined by

$$
\mathcal{T}_{\tau, \mu}[\mathrm{f}]:=\frac{\Gamma(\mu)}{\Gamma(\tau)} z^{1-\mu} \mathcal{D}_{z}^{\tau-\mu}\left[z^{\tau-1} \mathrm{f}\right],
$$

where $0<\tau \leq 1,0<\mu \leq 1,0 \leq \tau-\mu<1$ and $z \in \mathbb{U}$. As a result of a simple focus, the operator $\mathcal{D}_{z}^{\tau-\mu}[\cdot]$ is equivalent to the operator, which is the Srivastava-Owa operator of fractional derivative of order $\tau-\mu(0 \leq \tau-\mu<1)$ represented by (2). In special, it is clear that $\mathcal{T}_{1,1}[\mathrm{f}]=\mathrm{f}(\boldsymbol{z})$.

For our results specified by the Tremblay operator and fractional calculus, the following assertions will be required for both stating and proving.

Lemma 1 [5] Let the function $\mathrm{f}$ be in the class $\mathcal{A}(\mathrm{n})$. Then,

$$
\mathcal{T}_{\tau, \mu}[\mathrm{f}]=\frac{\tau}{\mu} z+\sum_{\mathrm{k}=\mathrm{n}+1}^{\infty} \frac{\Gamma(\mathrm{k}+\tau) \Gamma(\mu)}{\Gamma(k+\mu) \Gamma(\tau)} a_{k} z^{k},
$$

where $0<\tau \leq 1,0<\mu \leq 1,0 \leq \tau-\mu<1$ and $z \in \mathbb{U}$.

Lemma 2 [5] Let the function $\mathrm{f}$ be in the class $\mathcal{A}(\mathrm{n})$. Then,

$$
\frac{\mathrm{d}}{\mathrm{d} z}\left(\mathcal{T}_{\tau, \mu}[\mathrm{f}]\right)=\frac{\tau}{\mu}+\sum_{\mathrm{k}=\mathrm{n}+1}^{\infty} \frac{\mathrm{k} \Gamma(\mathrm{k}+\tau) \Gamma(\mu)}{\Gamma(\mathrm{k}+\mu) \Gamma(\tau)} \mathrm{a}_{\mathrm{k}} z^{\mathrm{k}-1},
$$

where $0<\tau \leq 1,0<\mu \leq 1,0 \leq \tau-\mu<1$ and $z \in \mathbb{U}$. 
Lemma 3 [13] Let $\mathrm{p}$ be (non-constant and) analytic function in the set $\mathbb{U}$ with $\mathrm{p}(0)=1$. If there exists a point $z_{0} \in \mathbb{U}$ such that

$$
\mathfrak{R e}(p(z))>0 \quad\left(|z|<\left|z_{0}\right|<1\right), \quad \mathfrak{R e}\left(p\left(z_{0}\right)\right)=0 \text { and } \mathrm{p}\left(z_{0}\right) \neq 0,
$$

then

$$
p\left(z_{0}\right)=i a \quad \text { and }\left.\quad \frac{z p^{\prime}(z)}{p(z)}\right|_{z=z_{0}}=i \frac{c}{2}\left(a+\frac{1}{a}\right),
$$

where $\mathrm{a} \in \mathbb{R}-\{0\}$ and $\mathrm{c} \in \mathbb{R}$ with $\mathrm{c} \geq 1$.

\section{Certain results and implications}

In this section, we shall state and then prove certain results in relation with both the Tremblay operator and some of its applications, which are given by the following theorems.

Theorem 1 If any one of the following inequalities:

$$
\mathfrak{R e}\left(\frac{z \frac{\mathrm{d}^{2}}{\mathrm{dz} z^{2}}\left(\mathcal{T}_{\tau, \mu}[\mathrm{f}]\right)}{\frac{\mathrm{d}}{\mathrm{d} z}\left(\mathcal{T}_{\tau, \mu}[\mathrm{f}]\right)}\right)>-\frac{\alpha}{2(1-\alpha)} \quad\left(0 \leq \alpha \leq \frac{1}{2}\right)
$$

and

$$
\Re e\left(\frac{z \frac{\mathrm{d}^{2}}{\mathrm{~d} z^{2}}\left(\mathcal{T}_{\tau, \mu}[\mathrm{f}]\right)}{\frac{\mathrm{d}}{\mathrm{d} z}\left(\mathcal{T}_{\tau, \mu}[\mathrm{f}]\right)}\right)>-\frac{1-\alpha}{2 \alpha} \quad\left(\frac{1}{2} \leq \alpha<1\right)
$$

is provided, then

$$
\mathfrak{R e}\left(\frac{\mathrm{d}}{\mathrm{d} z}\left(\mathcal{T}_{\tau, \mu}[\mathrm{f}]\right)\right)>\alpha \frac{\tau}{\mu}
$$

is also provided, where $0<\tau \leq 1,0<\mu \leq 1,0 \leq \tau-\mu<1,0 \leq \alpha<1, z \in \mathbb{U}$ and $\mathrm{f} \in \mathcal{A}$.

Proof. In consideration of (5) in Lemma 2, define a function $p(z)$ in the implicit form:

$$
\frac{\mathrm{d}}{\mathrm{d} z}\left(\mathcal{T}_{\tau, \mu}[\mathrm{f}]\right)=\frac{\tau}{\mu}[\alpha+(1-\alpha) \mathrm{p}(z)],
$$

where $0<\tau \leq 1,0<\mu \leq 1$ and $0 \leq \tau-\mu<1,0 \leq \alpha<1, f \in \mathcal{A}$ and $z \in \mathbb{U}$. It is clear that the function $p(z)$ satisfies the condition $p(0)=1$ in the hypothesis of Lemma 3. Then, it follows from (11) that

$$
z \frac{\mathrm{d}^{2}}{\mathrm{~d} z^{2}}\left(\mathcal{T}_{\tau, \mu}[\mathrm{f}]\right)=(1-\alpha) \frac{\tau}{\mu} z p^{\prime}(z) \quad(0 \leq \alpha<1 ; f \in \mathcal{A} ; z \in \mathbb{U}) .
$$


By combining (11) and (12), the following equality:

$$
\frac{z \frac{\mathrm{d}^{2}}{\mathrm{~d} z^{2}}\left(\mathcal{T}_{\tau, \mu}[\mathrm{f}]\right)}{\frac{\mathrm{d}}{\mathrm{d} z}\left(\mathcal{T}_{\tau, \mu}[\mathrm{f}]\right)}=\frac{(1-\alpha) z p^{\prime}(z)}{\alpha+(1-\alpha) \mathrm{p}(z)} \quad(0 \leq \alpha<1 ; f \in \mathcal{A} ; z \in \mathbb{U})
$$

is easily obtained, where, of course,

$$
\frac{\mathrm{d}}{\mathrm{d} z}\left(\mathcal{T}_{\tau, \mu}[\mathrm{f}]\right) \neq 0 \quad(\forall z \in \mathbb{U}) .
$$

Assume now that there exists a point $z_{0}$ belonging to $\mathbb{U}$, which satisfies the condition:

$$
\mathfrak{R e}\left(\mathrm{p}\left(z_{0}\right)\right)=0 \quad\left(z_{0} \in \mathbb{U} ; \mathrm{p}\left(z_{0}\right) \neq 0\right),
$$

indicated by (6) of Lemma 3. Then, by applying of the assertions of Lemma 3 , given in (7), which are

$$
\begin{gathered}
\mathfrak{R e}\left(\mathrm{p}\left(z_{0}\right)\right)=i a \text { and }\left.\frac{z p^{\prime}(z)}{p(z)}\right|_{z=z_{0}}=i \frac{c}{2}\left(a+\frac{1}{a}\right) \\
(a \neq 0 ; c \in \mathbb{R} ; c \geq 1),
\end{gathered}
$$

in the equation (13), the following equivalent equations:

$$
\begin{aligned}
\Re\left(\left.\frac{z \frac{\mathrm{d}^{2}}{\mathrm{~d} z^{2}}\left(\mathcal{T}_{\tau, \mu}[\mathrm{f}]\right)}{\frac{\mathrm{d}}{\mathrm{d} z}\left(\mathcal{T}_{\tau, \mu}[\mathrm{f}]\right)}\right|_{z:=z_{0}}\right) & =\Re e\left(\left.\frac{(1-\alpha) z p^{\prime}(z)}{\alpha+(1-\alpha) p(z)}\right|_{z:=z_{0}}\right) \\
& =\Re e\left(\left.\frac{z p^{\prime}(z)}{p(z)} \frac{(1-\alpha) p(z)}{\alpha+(1-\alpha) p(z)}\right|_{z:=z_{0}}\right) \\
& =-\frac{c}{2} \frac{\alpha(1-\alpha)\left(1+\mathrm{a}^{2}\right)}{\alpha^{2}+(1-\alpha)^{2} a^{2}}
\end{aligned}
$$

are easily obtained. By taking in consideration the following inequalities:

$$
\frac{1+a^{2}}{a^{2}+\left(\frac{\alpha}{1-\alpha}\right)^{2}} \geq 1 \text { if } 0 \leq \alpha \leq \frac{1}{2}
$$

and

$$
\frac{1+a^{2}}{1+\left(\frac{1-\alpha}{\alpha}\right)^{2} a^{2}} \geq 1 \text { if } \frac{1}{2} \leq \alpha \leq 1
$$


for (14), the inequality:

$$
\begin{aligned}
-\mathfrak{R e}\left(\left.\frac{z \frac{\mathrm{d}^{2}}{\mathrm{~d} z^{2}}\left(\mathcal{T}_{\tau, \mu}[\mathrm{f}]\right)}{\frac{\mathrm{d}}{\mathrm{d} z}\left(\mathcal{T}_{\tau, \mu}[\mathrm{f}]\right)}\right|_{z:=z_{0}}\right) & =\frac{c}{2} \frac{\alpha(1-\alpha)\left(1+\mathrm{a}^{2}\right)}{\alpha^{2}+(1-\alpha)^{2} \mathrm{a}^{2}} \\
& \geq\left\{\begin{array}{cl}
\frac{\alpha}{2(1-\alpha)} & \text { if } 0 \leq \alpha \leq \frac{1}{2} \\
\frac{1-\alpha}{2 \alpha} & \text { if } \frac{1}{2} \leq \alpha \leq 1
\end{array}\right.
\end{aligned}
$$

is also determined. But, the cases above are contradiction, respectively, with the inequalities given in (8) and (9). In this case, there is no any point $z_{0} \in \mathbb{U}$ satisfying the condition $\mathfrak{R} e\left(p\left(z_{0}\right)\right)=0$. This means that $\mathfrak{R e}(\mathrm{p}(z))>0$ for all $z$ in $\mathbb{U}$. Thus, (1) gives us the inequality given in (10). Therefore, this completes the proof of Theorem 1.

For the proofs of the following theorems (Theorems 2-4 below), under those important conditions:

$$
0<\tau \leq 1,0<\mu \leq 1,0 \leq \tau-\mu<1,0 \leq \alpha<1, z \in \mathbb{U} \text { and } \mathrm{f} \in \mathcal{A}(\mathrm{n}),
$$

in view of Lemma 1 or Lemma 2, and by taking into account the different definitions of the function $p(z)$ that we have defined just above, namely, in the proof of Theorem 1, when one defines that function $p(z)$ again, respectively, as in the following implicit forms:

$$
\begin{gathered}
\frac{\mathcal{T}_{\tau, \mu}[\mathrm{f}]}{z}=\frac{\tau}{\mu}[\alpha+(1-\alpha) \mathrm{p}(z)] \\
{\left[\frac{\mathrm{d}}{\mathrm{d} z}\left(\mathcal{T}_{\tau, \mu}[\mathrm{f}]\right)\right]^{\chi}=\left(\frac{\tau}{\mu}\right)^{x}[\alpha+(1-\alpha) p(z)]}
\end{gathered}
$$

and

$$
\left(\frac{\mathcal{T}_{\tau, \mu}[\mathrm{f}]}{z}\right)^{\chi}=\left(\frac{\tau}{\mu}\right)^{x}[\alpha+(1-\alpha) p(z)]
$$

and then follows the ways or steps used in the proof of Theorem 1, of course, by the help of Lemma 3, the following theorems (Theorems 2-4 below) can be easily proven. Their proofs are here omitted.

Theorem 2 If any one of the following inequalities:

$$
\mathfrak{R e}\left(\frac{z \frac{\mathrm{d}}{\mathrm{d} z}\left(\mathcal{T}_{\tau, \mu}[\mathrm{f}]\right)}{\mathcal{T}_{\tau, \mu}[\mathrm{f}]}\right)>\frac{2-3 \alpha}{2(1-\alpha)} \quad\left(0 \leq \alpha \leq \frac{1}{2}\right)
$$


and

$$
\mathfrak{R e}\left(\frac{z \frac{\mathrm{d}}{\mathrm{d} z}\left(\mathcal{T}_{\tau, \mu}[\mathrm{f}]\right)}{\mathcal{T}_{\tau, \mu}[\mathrm{f}]}\right)>\frac{3 \alpha-1}{2 \alpha} \quad\left(\frac{1}{2} \leq \alpha<1\right)
$$

is ensured, then

$$
\Re e\left(\frac{\mathcal{T}_{\tau, \mu}[\mathrm{f}]}{z}\right)>\alpha \frac{\tau}{\mu}
$$

is also ensured, where $0<\tau \leq 1,0<\mu \leq 1,0 \leq \tau-\mu<1,0 \leq \alpha<1, z \in \mathbb{U}$ and $\mathrm{f} \in \mathcal{A}$.

Theorem 3 If any one of the following inequalities:

$$
\mathfrak{R e}\left(\chi \cdot \frac{z \frac{\mathrm{d}^{2}}{\mathrm{~d} z^{2}}\left(\mathcal{T}_{\tau, \mu}[\mathrm{f}]\right)}{\frac{\mathrm{d}}{\mathrm{d} z}\left(\mathcal{T}_{\tau, \mu}[\mathrm{f}]\right)}\right)>-\frac{\alpha}{2(1-\alpha)} \quad\left(0 \leq \alpha \leq \frac{1}{2}\right)
$$

and

$$
\mathfrak{R e}\left(\chi \cdot \frac{z \frac{\mathrm{d}^{2}}{\mathrm{~d} z^{2}}\left(\mathcal{T}_{\tau, \mu}[\mathrm{f}]\right)}{\frac{\mathrm{d}}{\mathrm{d} z}\left(\mathcal{T}_{\tau, \mu}[\mathrm{f}]\right)}\right)>-\frac{1-\alpha}{2 \alpha} \quad\left(\frac{1}{2} \leq \alpha<1\right)
$$

is supplied, then

$$
\mathfrak{R e}\left[\left(\frac{\mathrm{d}}{\mathrm{d} z}\left(\mathcal{T}_{\tau, \mu}[\mathrm{f}]\right)\right)^{\mathrm{X}}\right]>\alpha\left(\frac{\tau}{\mu}\right)^{\chi}
$$

is also supplied, where $0<\tau \leq 1,0<\mu \leq 1,0 \leq \tau-\mu<1,0 \leq \alpha<1$, $\chi \in \mathbb{C}-\{0\}, z \in \mathbb{U}$, and $\mathrm{f} \in \mathcal{A}$ and also the value of the above complex power is taken to be its principal value.

Theorem 4 If any one of the following inequalities:

$$
\mathfrak{R e}\left(\chi \cdot \frac{z \frac{\mathrm{d}}{\mathrm{dz}}\left(\mathcal{T}_{\tau, \mu}[\mathrm{f}]\right)}{\mathcal{T}_{\tau, \mu}[\mathrm{f}]}\right)>\mathfrak{R e}(\chi)-\frac{\alpha}{2(1-\alpha)} \quad\left(0 \leq \alpha \leq \frac{1}{2}\right)
$$

and

$$
\mathfrak{R e}\left(\chi \cdot \frac{z \frac{\mathrm{d}}{\mathrm{d} z}\left(\mathcal{T}_{\tau, \mu}[\mathrm{f}]\right)}{\mathcal{T}_{\tau, \mu}[\mathrm{f}]}\right)>\mathfrak{R e}(\chi)-\frac{1-\alpha}{2 \alpha} \quad\left(\frac{1}{2} \leq \alpha<1\right)
$$

is verified, then

$$
\mathfrak{R e}\left[\left(\frac{\mathcal{T}_{\tau, \mu}[\mathrm{f}]}{z}\right)^{\chi}\right]>\alpha\left(\frac{\tau}{\mu}\right)^{\chi}
$$

is also verified, where $0<\tau \leq 1,0<\mu \leq 1,0 \leq \tau-\mu<1,0 \leq \alpha<1$, $\chi \in \mathbb{C}-\{0\}, z \in \mathbb{U}$, and $\mathrm{f} \in \mathcal{A}$ and also the value of the above complex power is taken to be its principal value. 
As certain implications of Theorems 1-4 above, when one centers on all theorems, it is easy to observe that they include several useful consequences of them, which relate to certain inequalities specified by the functions belonging to the class $\mathcal{A}(\mathrm{n})$. Particularly, some of them deal with the theory of (analytic and) univalent functions. For their details, see the works in [4] and [6]. As example and special consequences of them, we want to emphasize only two appertaining to the study of the relations between the analytic properties of a function $\mathrm{f}(z)$ in the class $\mathcal{A}(\mathrm{n})$ and the geometric properties of the image domain $f(\mathbb{U})$. One may see their details in [4], [6] and [8]-[12]. The others possible consequences, which are well-known in the literature and are also omitted here, are presented to reveal to the attention of the researchers who are interested in the topics of this work.

By setting $\tau:=\mu:=1$ in Theorem 1 , for a function $\mathrm{f}:=\mathrm{f}(z) \in \mathcal{A}(\mathrm{n})$, $f \equiv \mathcal{T}_{1,1}[\mathrm{f}]$ is then received. In the circumstances, as the first consequence of the main results, which is in relation with close-to-starlikeness of a function $\mathrm{f}$ in the class $\mathcal{A}(\mathrm{n})$, it can be obtained by the following-well-known result (Proposition 1 below).

Proposition 1 If a function $\mathrm{f} \in \mathcal{T}(\mathrm{n})$ satisfies any one of the cases in the following inequality:

$$
\mathfrak{R e}\left(\frac{z f^{\prime \prime}(z)}{f^{\prime}(z)}\right)>\left\{\begin{array}{cl}
-\frac{\alpha}{2(1-\alpha)} & \text { if } 0 \leq \alpha \leq \frac{1}{2} \\
-\frac{1-\alpha}{2 \alpha} & \text { if } \frac{1}{2} \leq \alpha \leq 1
\end{array},\right.
$$

then

$$
\mathfrak{R e}\left(f^{\prime}(z)\right)>\alpha \quad(0 \leq \alpha<1 ; z \in \mathbb{U}),
$$

that is, that $\mathrm{f}$ is a close-to-starlike function of order $\alpha$ in $\mathbb{U}$.

By setting $\tau:=\mu:=\chi:=1$ in Theorem 4, because of $\mathrm{f} \equiv \mathcal{T}_{1,1}[\mathrm{f}] \in \mathcal{A}(\mathrm{n})$, as the second consequence of the main results concerning close-to-convexity of a function $\mathrm{f} \in \mathcal{A}(\mathrm{n})$, it can be also revealed by the following result (Proposition 2 below).

Proposition 2 If a function $\mathrm{f} \in \mathcal{T}(\mathrm{n})$ satisfies any one of the cases in the following inequality:

$$
\mathfrak{R e}\left(\frac{z f^{\prime}(z)}{f(z)}\right)>\left\{\begin{array}{cl}
\frac{2-3 \alpha}{2(1-\alpha)} & \text { if } 0 \leq \alpha \leq \frac{1}{2} \\
\frac{3 \alpha-1}{2 \alpha} & \text { if } \quad \frac{1}{2} \leq \alpha \leq 1
\end{array},\right.
$$

then

$$
\mathfrak{R e}\left(\frac{\mathrm{f}(z)}{z}\right)>\alpha \quad(0 \leq \alpha<1 ; z \in \mathbb{U}),
$$

that is, that $\mathrm{f}$ is a close-to-convex function of order $\alpha$ in $\mathbb{U}$. 


\section{References}

[1] O. Altıntaş, H. Irmak, H. M. Srivastava, Fractional calculus and certain starlike functions with negative coefficients, Comput. Math. Appl., 30 (2) (1995), 9-15.

[2] M.P. Chen, H. Irmak, H. M. Srivastava, Some families of multivalently analytic functions with negative coefficients, J. Math. Anal. Appl., 214 (2) (1997), 674-690.

[3] M. P. Chen, H. Irmak, H. M. Srivastava, A certain subclass of analytic functions involving operators of fractional calculus, Comput. Math. Appl., 35 (2) (1998), 83-91.

[4] P. L. Duren, Grundlehren der Mathematischen Wissenchaffen, SpringerVerlag, New York, Berlin, Heidelberg, Tokyo, 1983.

[5] Z. Esa, H. M. Srivastava, A. Kılıçman, R.W. Ibrahim, A novel subclass of analytic functions specified by a family of fractional derivatives in the complex domain, Filomat, 31 (9) (2017), 2837-2849.

[6] A. W. Goodman, Univalent functions, Vols. I and II., Polygonal Publishing House, Washington-New Jersey, 1983.

[7] H. Irmak, Certain complex equations and some of their implications in relation with normalized analytic functions, Filomat, 30 (12) (2016), 33713376.

[8] H. Irmak, Some novel applications in relation with certain equations and inequalities in the complex plane, Math. Commun., 23 (1) (2018), 9-14.

[9] H. Irmak, B. A. Frasin, An application of fractional calculus and its implications relating to certain analytic functions and complex equations, J. Fract. Calc. Appl., 6 (2) (2015), 94-100.

[10] H. Irmak, P. Agarwal, Some comprehensive inequalities consisting of Mittag-Leffler type functions in the complex plane, Math. Model. Nat. Phenom., 12 (3) (2017) 65-71.

[11] H. Irmak, P. Agarwal, Comprehensive Inequalities and Equations Specified by the Mittag-Leffler Functions and Fractional Calculus in the Complex Plane. In: Agarwal P., Dragomir S., Jleli M., Samet B. (eds) Advances 
in Mathematical Inequalities and Applications. Trends in Mathematics. Birkhäuser, Singapore, 2018.

[12] M. Şan, H. Irmak, A note on some relations between certain inequalities and normalized analytic functions, Acta Univ. Sapientiae, Math., 10 (2) (2018), 368-374.

[13] M. Nunokawa, On properties of non-Caratheodory functions, Proc. Japan Acad. Ser. A Math. Sci., 68 (6) (1992), 152-153.

[14] S. Owa, On the distortion theorems. I, Kyungpook Math. J., 18 (1) (1978), $53-59$.

[15] H. M. Srivastava, S. Owa (Editors), Univalent Functions, Fractional Calculus and Their Applications, Halsted Press, John Wiley and Sons. New york, Chieschester, Brisbane, Toronto, 1989.

[16] R. W. Ibrahim, J. M. Jahangiri, Boundary fractional differential equation in a complex domain, Boundary Value Prob. 2014, Article ID 66, pp. $1-11,2014$. 